\title{
Measurement of unsteady loading and power output variability in a micro wind farm model in a wind tunnel
}

\author{
Juliaan Bossuyt - Michael F. Howland • Charles Meneveau • Johan \\ Meyers
}

Received: date / Accepted: date

\begin{abstract}
Unsteady-loading and spatio-temporal characteristics of power output are measured in a windtunnel experiment of a micro-scale wind farm model with 100 porous disk models. The model wind farm is placed in a scaled turbulent boundary layer and six different layouts, varied from aligned to staggered, are considered. The measurements are done by making use of a specially designed small-scale porous disk model, instrumented with strain gages. The frequency response of the measurements goes up to the natural frequency of the model, which corresponds to a reduced frequency of 0.6 when normalized by the diameter and the mean hub height velocity. The equivalent range of time-scales, scaled to field-scale values, is 15 seconds and longer. The accuracy and limitations of the acquisition technique are documented and verified with hot-wire measurements. The spatio-temporal measurement capabilities of the experimental setup are used to study the cross-correlation in the power output of various porous
\end{abstract}

J. Bossuyt

KU Leuven, Department of Mechanical Engineering, Celestijnenlaan 300, Leuven, Belgium.

Tel.: +123-45-678910

E-mail: juliaan.bossuyt@kuleuven.com

M. F. Howland

Department of Mechanical Engineering, Johns Hopkins University, 3400 North Charles Street, Baltimore, MD21218, USA.

\section{Meneveau}

Department of Mechanical Engineering and Center for Environmental and applied Mechanics, Johns Hopkins University, 3400 North Charles Street, Baltimore, MD21218, USA.

J. Meyers

KU Leuven, Department of Mechanical Engineering, Celestijnenlaan 300, Leuven, Belgium. disk models of wind turbines. A significant correlation is confirmed between streamwise aligned models, while staggered models show an anti-correlation.

Keywords wind tunnel measurements - wind farm . porous disk model · spatio-temporal · correlations · unsteady loading · power output variability

\section{Introduction}

Variability in the power output of wind turbines introduces a challenge for the integration of large amounts of wind energy in an electricity grid. Power output variability is inherently present due to the unsteady characteristics of the atmospheric boundary layer, from which the turbines extract kinetic energy. Velocity fluctuations are present on many time scales resulting from turbulence, and on larger scales from e.g. meso-scale, diurnal, synoptic or seasonal variations. Changing conditions furthermore cause unsteady loading of the wind turbine components. The necessary fill-in power to compensate for the power output variability and the need for stronger components increase the total cost of wind energy $[27,43,21,19]$. Therefore, an improved knowledge of the interaction between the unsteady boundary layer and the wind turbines in a wind farm is necessary.

The variance of the mean of $N$ independent identically distributed signals reduces with $1 / N$ according to: $\operatorname{Var}(\bar{x})=\overline{\operatorname{Var}\left(x_{i}\right)} / N$. It can thus be expected that the power fluctuations of energy from wind can be reduced by summation over multiple wind turbines or for example an entire wind farm. Field data [2] and later also a large-eddy simulation (LES) study [47] have shown that the reduction of the power fluctuations, from averaging over a large number of turbines, results in a non-trivial 
reduction compared to what can be expected for independent identically distributed signals. More specifically, the fluctuations of the total power output of many wind turbines have been observed to follow a power law behavior similar to a Kolmogorov scaling with a slope close to $-5 / 3[2,47]$. It is suggested by several papers $[47,44]$ that the strong streamwise correlation in the boundary layer might play an important role in this non-trivial variability reduction. However, the mechanism causing such a behavior in the power spectrum has not been clearly explained.

To this end, wind tunnel measurements can bring additional insight. They have the advantage over fullscale experiments that boundary conditions can be carefully controlled. An important challenge for studying power output variability and unsteady loading in a wind tunnel is performing measurements with sufficiently high temporal and spatial resolution for a turbine array containing a large number of model turbines.

A number of experimental wind-tunnel studies have focused on detailed flow measurements for small wind turbine arrays $[24,31,9,30,7]$. Others have studied larger wind farms and their interaction with the boundary layer $[39,12,20,48]$. Flow measurements typically focus on single point hot-wire measurements with a high temporal resolution or PIV measurements, which focus on smaller subdomains of the flow field. Theunissen et al. [48] have used a measurement set-up with 80 porous disk models to study the influence of wind direction on the overall temporal-mean power output. In the current study we make use of a similar porous disk approach to perform measurements of the spatio-temporal power output characteristics. To this end, each model is instrumented with a strain gage, and known relations between bending moment, thrust, velocity and power output are used to interpret the data. The design and instrumentation of the porous disk model is described in $\S 2$. The measurement capabilities are demonstrated in $\S 3$ with wind tunnel experiments of a micro-scale wind farm consisting of 100 porous disk models. Average row power, unsteady loading and spatio-temporal correlations are studied for six different layouts, varied gradually from fully aligned to fully staggered.

\section{Experimental set-up}

In this study we make use of the Corrsin Wind Tunnel at the Johns Hopkins University. This closed loop facility has a test section of $0.9 \mathrm{~m} \times 1.2 \mathrm{~m}$ and an approximate length of $10 \mathrm{~m}$. The cross-section increases downstream to compensate for boundary layer development along the walls. A primary contraction-ratio of $25: 1$ and a secondary of 1.27:1 result in a smooth inflow

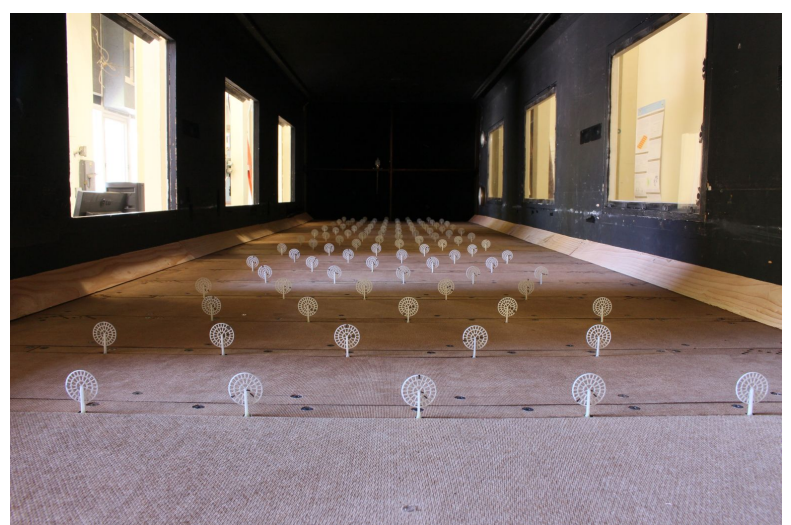

Fig. 1 Photograph of the micro wind farm set-up in the wind tunnel.

at the beginning of the test section with a background turbulence intensity of $T I_{u} \approx 0.12 \%$. Either an active grid, passive grid or a developed turbulent boundary layer are used to generate desired flow conditions (cf. further discussion in next sections).

An experimental study of a wind farm with a large number of turbines in a wind tunnel of limited size requires the design of a small-scale wind-turbine model. In the current work, we aim at fitting 100 turbine models in the wind tunnel with typically realistic spacings. The intended wind farm set-up, is illustrated in figures 1,2 and 3. It allows for a fast and easy adaptation of the turbine arrangements (cf. figure 3), which is exploited to study power variability for different wind farm layouts. Details are further discussed in $\S 3$.

Studying the power output of a wind farm in a windtunnel experiment requires a model that accurately reproduces the turbine wake, and also allows to correctly measure an appropriate surrogate for power output [48]. Based on these two main requirements the choice and design of a suitable model is described in $\S 2.1$. The measured wake characteristics are verified with results in the literature in $\S 2.2$. The designed models are instrumented with strain gages, allowing thrust (as a surrogate of power) measurements as described in $\S 2.3$. The related acquisition and signal reconstruction technique are described in $\S 2.4$ and compared to a single point hot-wire measurement. This section concludes with the calibration procedure of the porous disk models in $\S 2.5$.

\subsection{Design of a model for a scaled wind turbine model}

A first requirement for a scaled wind-turbine representation is a correct characterization of the wake structure [48]. A possible approach is based on geometric scaling of the turbine design $[9,36,41,33,34,15,53,28,30,7$, $12,20,56]$. However, our interest is in placing a large 


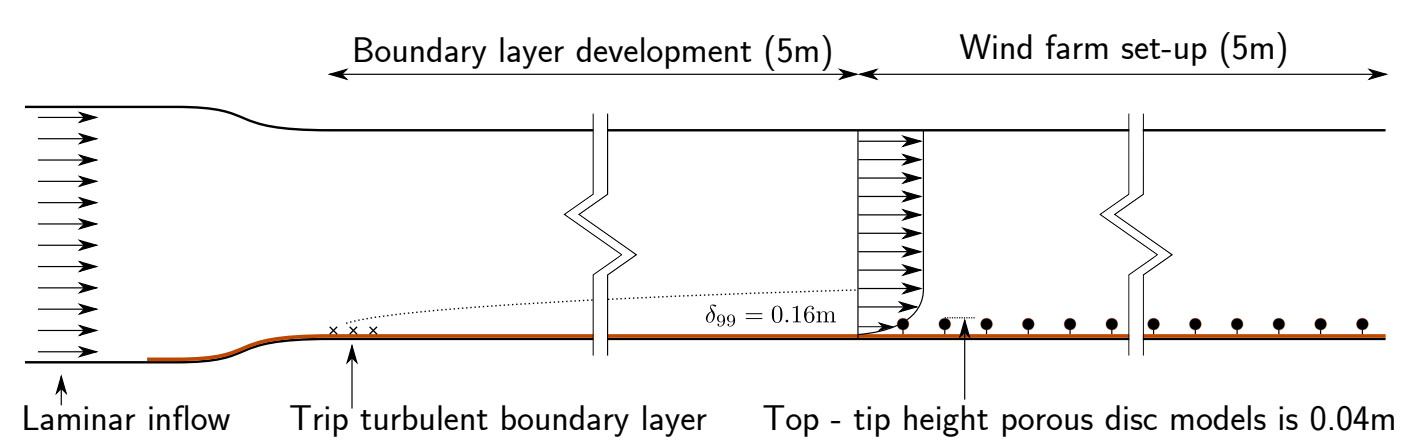

Fig. 2 Schematic representation of the measurement set-up in the Corrsin wind tunnel.

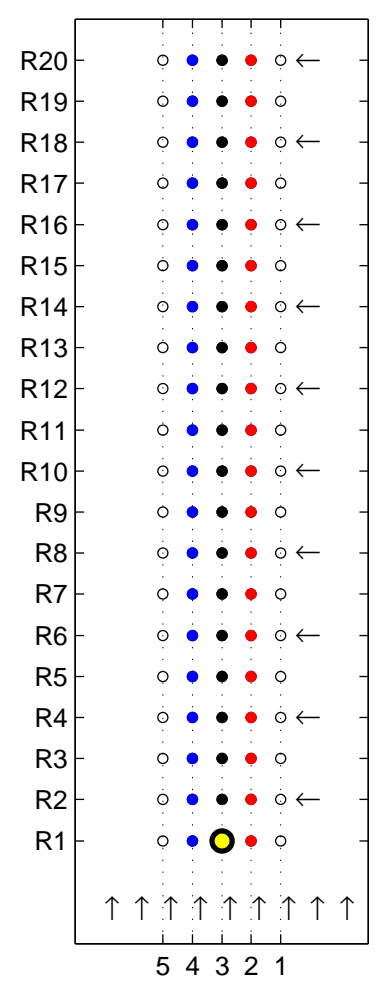

Fig. 3 Schematic representation of the model wind farm layout with 5 columns in the streamwise direction and 20 rows in the spanwise direction. Horizontal arrows indicate rows which can be shifted to change the layout.

number of turbines in the wind tunnel, which dictates a model rotor diameter of $3 \mathrm{~cm}$. Using rotating blades for such small rotors, and building and operating 100 of them in the wind tunnel is not practical. Moreover, scaled rotating wind turbine models have inherent limitations since perfect flow similarity is not possible due to large scale differences.

A second approach to building a scaled turbine model is the use of a porous disk. Such a porous disk is a momentum sink that does not directly extract energy from the flow, but dissipates kinetic energy instead by generating small scale turbulence in the near wake of the disk [34]. Wind turbines can be considered porous media by their significant amount of flow-through. Porous disk models have proven useful in reproducing approximate wind turbine wakes in wind tunnel experiments $[32,3$, $17,48]$, and in numerical simulations that use the actuator disk representation $[38,54,14,46,47]$. The near-wake of a porous disk differs as turbulence is produced by a grid, while turbines introduce rotational momentum, tip and hub vortices and turbulence from the blades [56]. However, blade signatures and rotational momentum have shown to be overshadowed by ambient velocity fluctuations in the far wake [3]. It is found that the main source of turbulence production in the far wake, where the flow becomes self-similar, is velocity shear [3]. By matching the self-similar velocity profile of a windturbine wake, porous disk models can create a similar far wake as rotating wind turbine models in case of turbulent flow conditions $[32,3,17,48,10]$. Furthermore, a comparison study [10] of the flow field in the wake of a porous disk and a rotor model has shown that transport of mean kinetic energy is adequately represented in areas where the rotation of the wake is not a critical phenomenon. Located in a turbulent boundary layer, the region where wake rotation is significant stays limited to the near wake of the wind turbine. This makes porous disk models useful to study the power output of a large wind farm, when focusing on physical phenomena occurring on length and time scales larger than those corresponding to the rotor diameter.

Next to a correct wake characterization, a second requirement for a scaled wind turbine model is the measurement of the thrust force with a sufficiently high frequency response. Fluctuations in the power output are present on many time scales, due to turbulence, large weather phenomena or at higher frequencies due to for example the blade-tower passage. As a wind turbine spatially filters the turbulent flow field over its swept area, the effect of small scale turbulence on the total power output variability is reduced [18]. In this study we focus on the power output variability generated by the larger energy-containing turbulent eddies, larger than the wind turbine diameter. The relevant 


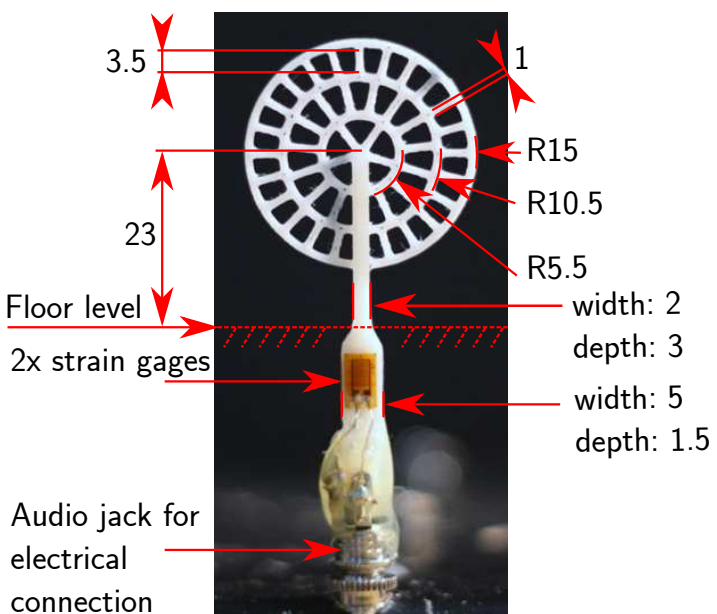

Fig. 4 Photograph of the porous disk model. Dimensions are given in $\mathrm{mm}$

time scales in full scale wind farms range from tens of seconds to hours.

Given the geometric scaling factor of 1:3333 (compared to a full scale wind turbine with a diameter of $100 \mathrm{~m}$ ), and wind-tunnel velocities that remain in the same range of full scale conditions, equivalent windtunnel time scales are 3333 times faster. In order to capture these dynamical timescales of interest, an acquisition frequency up to a few hundred Hertz is required for the wind tunnel experiments.

Full-scale wind turbines operating in the below-rated region (often referred to as region 2) are controlled to maximize aerodynamic efficiency by adapting the rotor speed and pitch angle to the incoming wind velocity [1]. In this regime, the overall turbine thrust coefficient is roughly constant, typically in the range $C_{T}=0.75-0.85$. It is relatively simple to match such a thrust coefficient with a porous disk, with a value that is only weakly dependent on Reynolds number [40], so that it remains roughly constant for a range of wind-tunnel velocities. Hence, a properly designed porous disk model can match the control of a turbine working in region 2. This approach is commonly used to represent wind turbines in large-eddy simulations of large wind farms using actuator disk models on a relatively coarse grid $[38,54,14,46,47]$. In contrast, the rated regime (region 3 ) also occurs less frequently and there is less potential for improvements as the turbines already operate at their maximum power output. In this study we focus on the below-rated regime.

Given the considerations discussed above, we selected to use porous disk models in the current study. The final design of the model is shown in figure 4 . The porous disk was designed to match a realistic turbine thrust coefficient while keeping the solidity low.

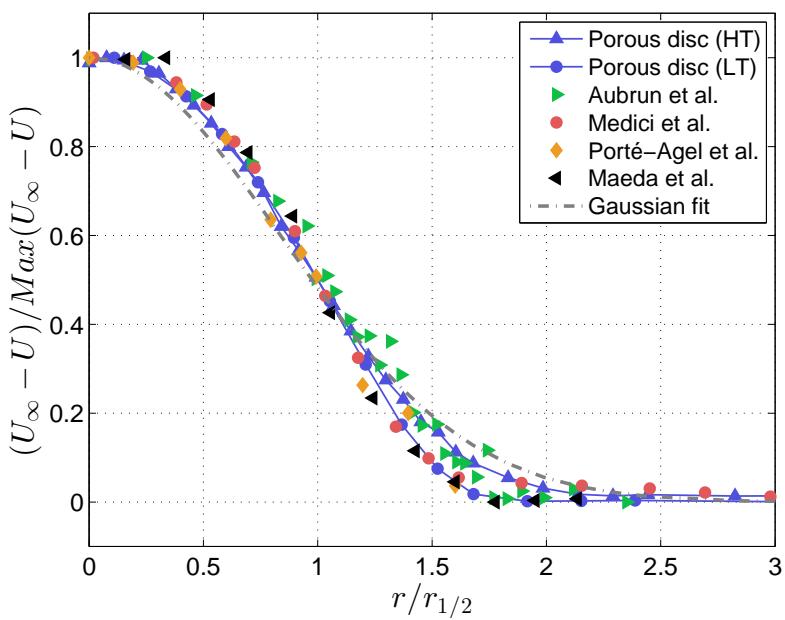

Fig. 5 Wake measurement of the normalized steamwise velocity deficit at a downstream distance of $x / D=3$. Turbine models are documented in table 1.

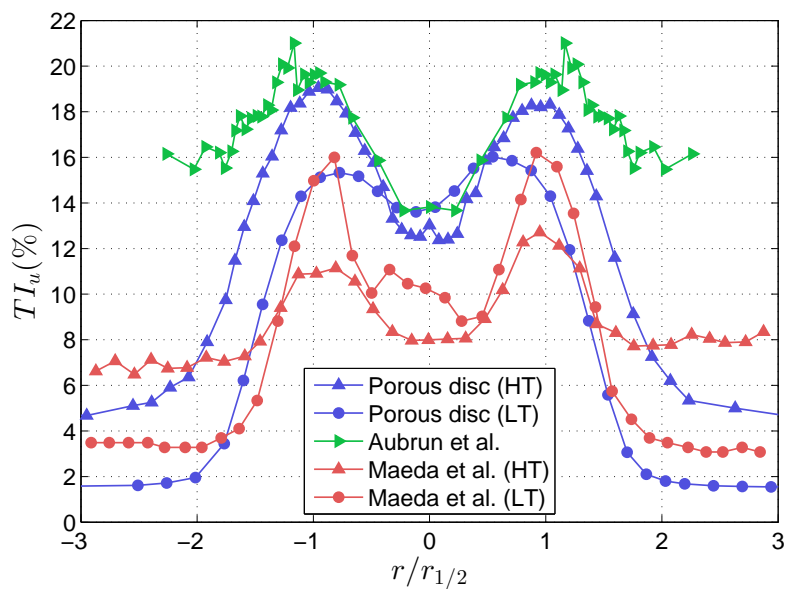

Fig. 6 Wake measurement of the streamwise turbulence intensity at a downstream distance of $x / D=3$. Turbine models are documented in table 1.

The final design has a measured thrust coefficient of $C_{T}=0.75 \pm 0.04$ and a solidity of $\beta=0.5$. The thrust coefficient was measured in a uniform flow with a background turbulence intensity of $T I_{u}=0.12 \%$ by means of strain gages on the model tower (cf. $\$ 2.3$ for details on the strain-gage measurements).

\subsection{Wake measurements and comparison}

In order to measure the model wake characteristics, a porous disk is tested in the Corrsin wind tunnel, using a uniform inflow (the model is mounted sufficiently far from the wall). Wake measurements are performed with an in-house built 2-component hot-wire probe and a computer controlled three-axis traversing system. Data at each point are acquired for 26 seconds with a sampling frequency of $10 \mathrm{kHz}$ and a low pass filter of $5 \mathrm{kHz}$. 
Table 1 Characteristics of rotor wind turbine models from the literature used for the comparison in figure 5 and 6.

\begin{tabular}{|c|c|c|c|c|c|}
\hline & Porous disk & Aubrun et al. [3] & Medici et al. [36] & Chamorro et al [11] & Maeda et al [35] \\
\hline$C_{T}$ & $0.75 \pm 0.04$ & 0.5 & 0.84 & 0.42 & - \\
\hline$T I_{u}$ & $1.5 \%(\mathrm{LT})$ and $5 \%(\mathrm{HT})$ & $13 \%$ & $4.5 \%$ & $2 \%$ & $3 \%(\mathrm{LT})$ and $8 \%(\mathrm{HT})$ \\
\hline Diameter & $0.03 \mathrm{~m}$ & $0.416 \mathrm{~m}$ & $0.18 m$ & $0.13 \mathrm{~m}$ & $0.5 \mathrm{~m}$ \\
\hline
\end{tabular}

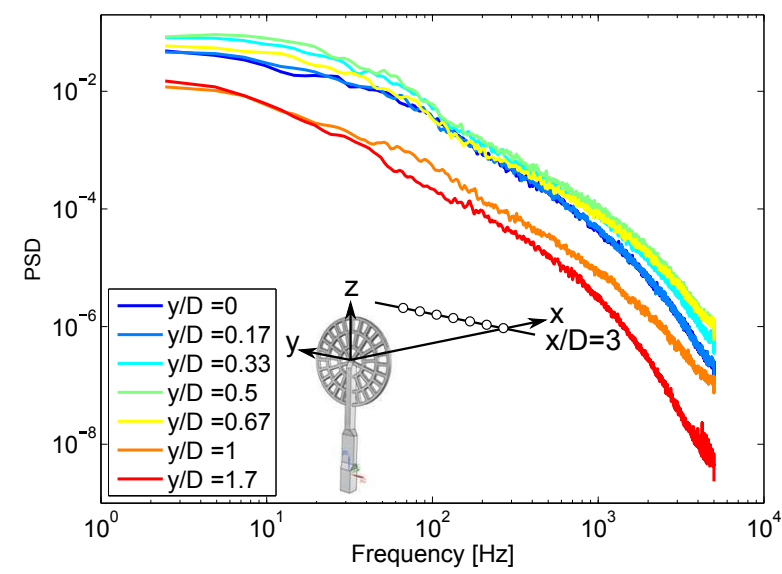

Fig. 7 One-dimensional spectrum of the streamwise velocity in the wake of the porous disk for the higher turbulence case (HT) at $x / D=3$ and at various cross-stream positions, shown as open circles.

The uncertainty on the velocity measurements is estimated to be less than $2 \%[50,26]$ and the uncertainty of the traversing system is estimated to be less than $\pm 0.1 \mathrm{~mm}$.

Horizontal profiles are measured at a downstream distance of $x / D=3$, because of the available data in the literature for this downstream location. The normalized mean streamwise velocity defect and turbulence intensity are shown in figures 5 and 6 respectively, and compared to results for rotating wind turbine models from the literature $[3,36,56,11,35]$ - see Table 1 for a list. Inflows with two different background turbulence intensity levels are considered. The higher turbulence case (HT) is generated with an active grid [49]. The lower turbulence case (LT) is generated with a passive grid with a mesh size of $0.1 \mathrm{~m}$ and a bar-width of $0.02 \mathrm{~m}$. The measured integral length scale, calculated from the area under the measured autocorrelation function to the first zero-crossing for the streamwise velocity and by making use of Taylor's hypothesis, is approximately $0.15 \mathrm{~m}$ for the active grid, and $0.07 \mathrm{~m}$ for the passive grid.

Figure 5 shows the scaled dimensionless velocity profiles, each normalized by the maximum velocity defect and half-wake thickness at $x / D=3$. Results indicate very good agreement among the measurements for dif- ferent types of turbine models. At $x / D=3$ the data are slightly less smeared out than the self-similar Gaussian profile that can be expected in the far wake. Discrepancies between the streamwise turbulence intensity profiles in figure 6 originate from differences in the ambient flow characteristics and the thrust coefficients. However, a qualitatively similar shape is seen for all profiles. This suggests that velocity shear is the main contributor for the turbulence production in the far wake, allowing the porous disk to match profile shapes from rotor models. It is seen that at a distance of only $x / D=3$ already a good agreement is found between the measured profiles and those in the literature for rotor models. It is expected that this agreement will improve further downstream, as blade signatures keep reducing and the wakes become more self-similar.

Finally, figure 7 shows the power spectrum of the streamwise velocity in the wake of the porous disk, at different spanwise locations. The absence of a distinct peak in the spectrum further confirms that our porous disk model does not display significant bluff body vortex shedding. Overall, it is concluded that the porous disk model is capable of reproducing the velocity and turbulence intensity profile in the far wake, with sufficient accuracy to allow studying the power output of a wind farm, focusing on length and time scales larger than those corresponding to several rotor diameters, and away from the near-wake region

\subsection{Model instrumentation}

By making use of the known thrust coefficient and measuring the thrust force, it is possible to estimate the incoming velocity at the porous disk model in a windfarm setup. The equivalent wind turbine power is then further estimated using standard relations from momentum theory. The total thrust force is determined by measuring the bending of the model tower with a strain gage apparatus. An Omega SGD-3/350-LY11 strain gage is attached to each side of the tower (see figures 4 and 8) and measured in a half-bridge configuration with an Omega iNET-423 and iNET-555 acquisition device. Making use of the i512 wiring box, no external bridge resistors are needed. 


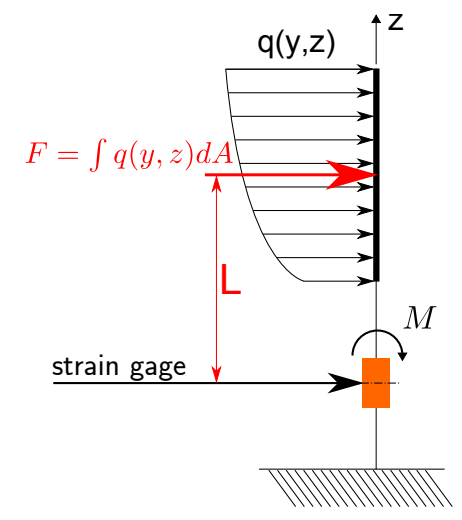

Fig. 8 Schematic representation of the force distribution on the disk, resulting in the bending-strain measured by the strain gage apparatus.

The measured strain depends linearly on the resulting bending moment acting at the location of the strain gage, assuming constant material properties (figure 8). For uniform load distributions and presuming a static situation, the bending moment corresponds to the total thrust force times the distance between the center of the disk and the strain gage $L$, with $q(y, z)$ the load per unit area and $F=\langle q\rangle A$ :

$M=\int_{A} q(y, z) z d A=\langle q\rangle\langle z\rangle A=F L$.

However, for non-uniform load distributions, the center of the force will shift away from the center of the disk (cf. figure 8), and is not known during the measurements. This effect is considered by decomposing the force distribution in a spatially averaged and spatially fluctuating part.

$q(y, z)=\langle q\rangle+\widetilde{q}(y, z)$

$M=\langle q\rangle\langle z\rangle A+\int_{A} \widetilde{q}(y, z) z d A$

The second term of the static bending moment relation represents the expected mean measurement error due to shear in the mean velocity profile. This static offset is estimated to be the order of $+4 \%$ by using the incoming mean velocity profile and assuming a constant thrust coefficient over the disk. Models more downstream can see a more uniform velocity profile due to wake mixing [12], and hence a smaller offset. Because the mean row power estimates are normalized by the estimated power in the first row, this static offset can lead to a lower power estimate for the downstream rows.

In a dynamic load situation, in which the thrust force fluctuates with the turbulent velocity field, the relation between measured bending moment, by the strain gage, and thrust on the disk is more complicated. By modeling the structural response of the model as a harmonic oscillator using its first and dominant natural frequency, the dynamic thrust force behavior can be reconstructed from the strain measurements. The steps in the reconstruction procedure are shown in figure 9. To this end, the natural frequency $f_{n}$, spring $k$ and damping $\zeta$ coefficients are determined for every porous disk model from a static and dynamic calibration (cf. $\S 2.5$ for details), allowing us to obtain measured values of the thrust force $F(t)$ from the measured strain time signal. With the thrust coefficient, the incoming spatially averaged velocity signal $\langle U\rangle(t)$ is reconstructed based on $F(t)=\rho\langle U\rangle^{2}(t) C_{T} A / 2$, where $A=\pi D^{2} / 4$ is the rotor area. The velocity represents a uniform incoming velocity which would result in the same thrust force as measured. The reconstructed velocity allows to estimate the equivalent power signal $P(t)=\rho\langle U\rangle^{3}(t) C_{P} A / 2$ of the model by assuming a realistic power coefficient. Note that the results shown in this paper on the ratio of power to the power of the first row are independent of the actual power coefficient.

\subsection{Frequency response analysis}

The accuracy of important parts of the reconstruction scheme is verified by comparing the reconstructed velocity $\langle U\rangle(t)$ from a porous disk with the simultaneously measured velocity from a hot-wire probe (figure 10). The verification is performed for a model in the wind farm, here shown for row 8 and column 2 of the staggered wind farm layout. The measurement setup is shown in figure 10. The streamwise velocity was measured with a one-component hot-wire probe $30 \mathrm{~mm}$ upstream from the porous disk center. Both the hot-wire and strain signals were sampled at a frequency of $10 \mathrm{kHz}$ and filtered by an analog low-pass filter at the Nyquist frequency or lower $(5 \mathrm{kHz}$ for the hot-wire probe and 4 $\mathrm{kHz}$ for the strain gage apparatus).

Figure 11 shows the power spectral density of the measured and reconstructed signals. The measured strain signal is shown in green and shows a clear peak at the natural frequency of the porous disk model. Frequencies above $270 \mathrm{~Hz}$ are not considered for the strain measurements, to ensure a sufficient signal to noise ratio. The power spectrum of the reconstructed thrust signal is shown in red, where the structural response model has compensated for the peak due to the natural frequency. The natural frequency is approximately $200 \mathrm{~Hz}$ and compares in this measurement to a reduced frequency of 0.6 , when normalized by the mean hub height velocity and disk diameter. It can thus be expected that the signature of the spatial filtering by the porous disk can be captured in the measurements, i.e. the thrust force and related velocity follow from an integral over 


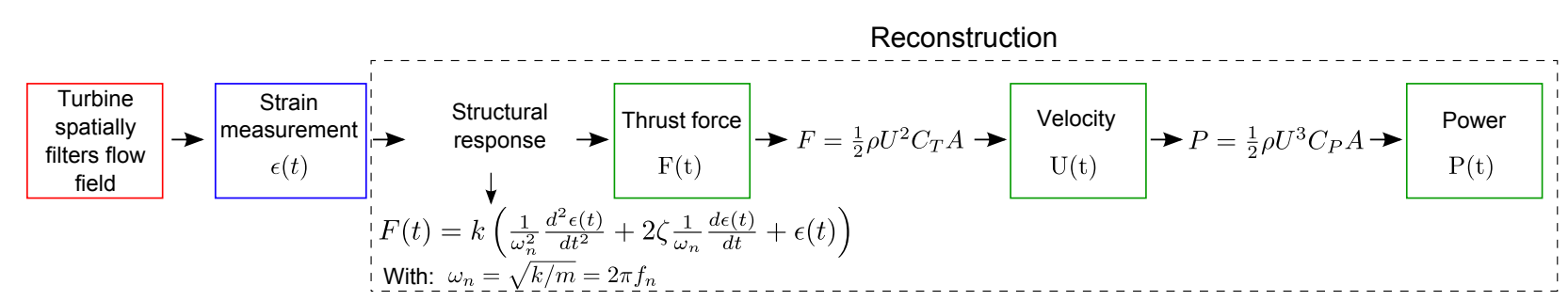

Fig. 9 Reconstruction procedure to estimate the power output from the strain gage measurement.

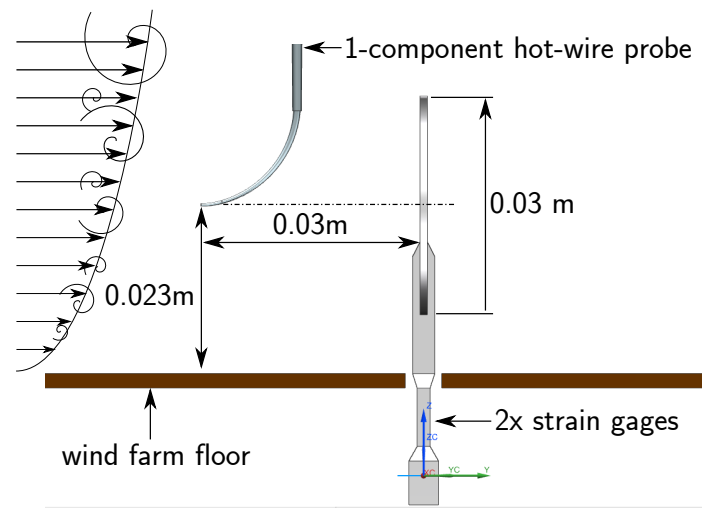

Fig. 10 Measurement set-up for the verification of the reconstruction procedure shown on figure 9 , for a porous disk model in the wind farm.

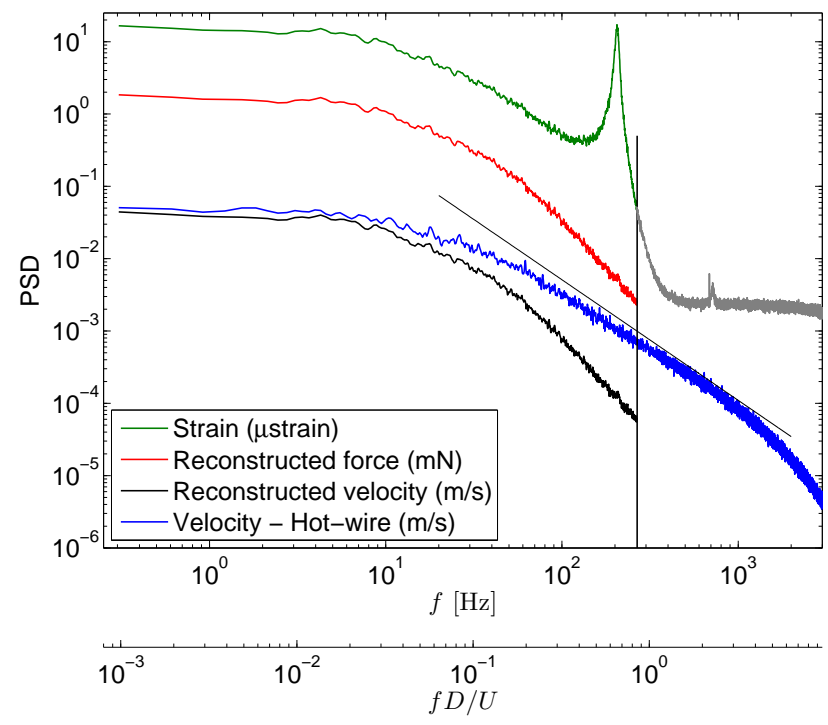

Fig. 11 Power spectral density of the measured and reconstructed signals for a porous disk model in row 8 and column 2 in a staggered wind farm case. A second $\mathrm{x}$-axis is shown with the frequency normalized by the mean hub-height velocity and the diameter of the model.

the disk area (see figure 8), and are thus not corresponding to a point measurement. Comparing the spectrum of the reconstructed velocity (shown in black) with the spectrum of the hot-wire velocity (shown in blue), a difference is indeed observed, consistent with the effect of a spatial filter. An attempt to analytically describe

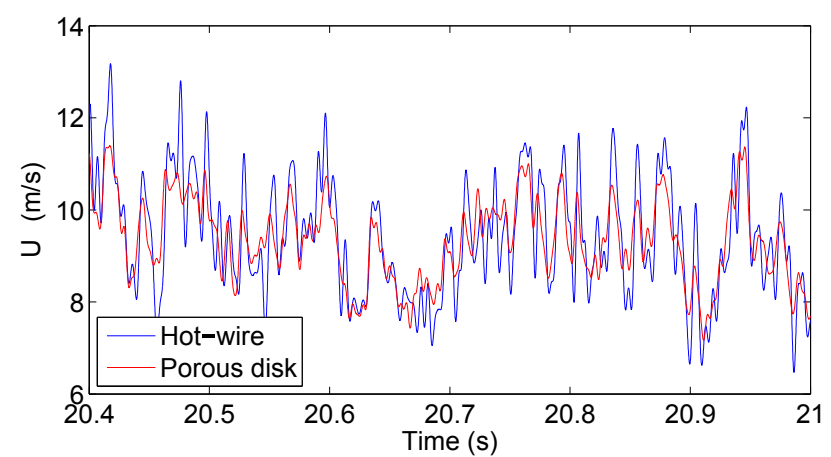

Fig. 12 Comparison of the reconstructed velocity from the porous disk in row 8 and column 2 in a staggered wind farm and the velocity from the hot-wire probe. Both signals are filtered at $f_{c}=200 \mathrm{~Hz}$. The correlation coefficient between both signals is $r=0.86$.

the spatial filtering is further discussed in the appendix. This type of spatial filtering is also present in full scale wind turbines. Overall, considering the spatial filtering, the porous disk model features a frequency response up to approximately $200 \mathrm{~Hz}$ or a reduced frequency of 0.6 in the wind farm measurements described here.

Figure 12 shows the measured hot-wire velocity and the reconstructed velocity from the porous disk. Both signals are filtered by a digital sharp cut-off filter with $f_{c}=200 \mathrm{~Hz}$ for the comparison. The qualitative comparison of both time signals in figure 12 shows a relatively good agreement. Differences are attributed to the difference in location between the hot-wire probe and the porous disk, and to the effect of spatial filtering by the porous disk. The correlation coefficient between both signals is $r=0.86$.

\subsection{Model turbine calibrations}

The reconstruction approach discussed above requires four physical calibration constants for each model: the spring constant $k$, damping constant $\zeta$, natural frequency $f_{n}$ and thrust coefficient $C_{T}$. Porous disk models were manufactured with $3 \mathrm{D}$ printing to guarantee the best possible accuracy. However, to account for the small changes in the material properties and the man- 


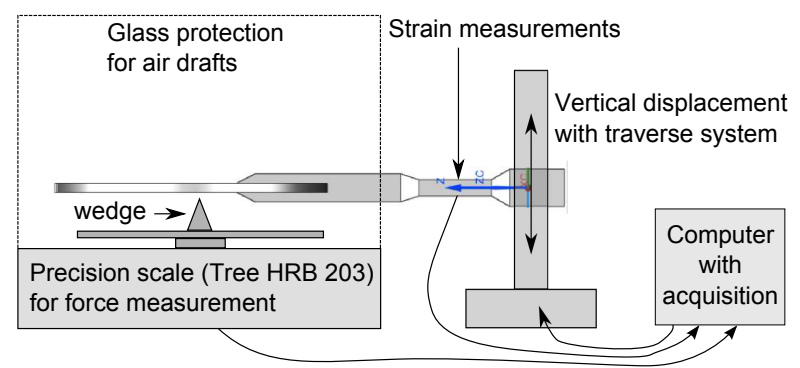

Fig. 13 Static calibration set-up. The precision scale and vertical traverse are computer controlled for an automatic calibration procedure.

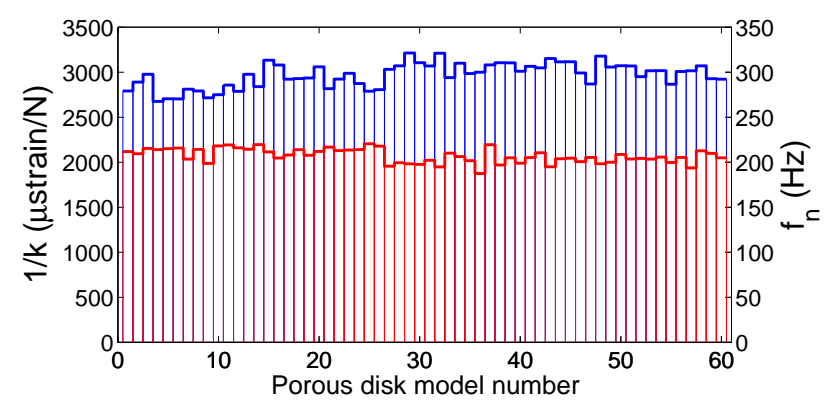

Fig. 14 The distribution of the measured spring constants (blue line) with a measurement uncertainty of $\delta_{k}= \pm 4 \%$ and the natural frequency (red line) for the selected porous disk models.

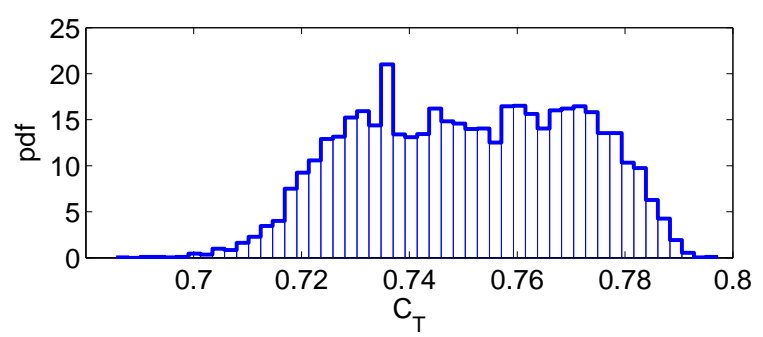

Fig. 15 The distribution of the measured thrust coefficient with $C_{T}=0.75 \pm 0.04$.

ual strain gage attachment-process, every model was calibrated separately.

The spring constant is determined from a static calibration, giving the relation between the force on the disk and the measured strain. An apparatus for this static calibration was designed for high accuracy and to facilitate the calibration of a large number of models (see figure 13). A force is applied by moving the porous disk vertically in small steps of approximately $0.02 \mathrm{~mm}$, while a wedge applies the force on the center of the disk. The wedge is located on a precision scale (Tree HRB 203), which allows the measurement of the force. To avoid the effect of air drafts on the force, the air volume around the porous disk is closed. The force and strain measurements are controlled together with the vertical traverse system. The measurement uncer- tainties are estimated to be smaller than $\pm 0.5 \%$ for the force and $\pm 1-4 \%$ for the strain, depending on the sampling rate and sample integration time. The precision of the traverse system has no direct influence on the calibration procedure. Through an error propagation analysis, the uncertainty of the spring constant is found to be $\delta_{k}= \pm 4 \%$.

The damping coefficient is measured from the impulse response. Within high accuracy, it was found to be the same for every model, so a single value $\zeta=0.03$ is taken. The natural frequency could in principle also be determined from the impulse response. However, it can also be computed from the peak in the spectrum of the strain signal, and as a result is inherently measured during every wind farm measurement. The average natural frequency is $\overline{f_{n}} \approx 200 \mathrm{~Hz}$. The measured spring constants and natural frequencies of the models selected for the measurements are shown in figure 14 .

Finally, the thrust coefficient was measured for all models by a simultaneous measurement of the strain from the porous disk and the velocity from a Pitot probe. The porous disk models were located in a uniform flow with a turbulence intensity of $T I_{u}=0.12 \%$. The thrust coefficient is averaged over three measurements for each model. Each measurement was performed for 5 minutes, covering a velocity range from $6 \mathrm{~m} / \mathrm{s}$ up to $12 \mathrm{~m} / \mathrm{s}$ to verify the Reynolds dependency. As every porous disk is designed with exactly the same shape, variations in the measured thrust coefficient at a constant velocity are expected to be related to manufacturing uncertainties, while variations as function of velocity are expected to be directly related to Reynolds number dependencies. We observed that from $6 \mathrm{~m} / \mathrm{s}$ to $7.5 \mathrm{~m} / \mathrm{s}$, the thrust coefficient increases from $C_{T} \approx 0.6$ to $C_{T} \approx 0.74$. Between $7.5 \mathrm{~m} / \mathrm{s}$ and $12 \mathrm{~m} / \mathrm{s}$ the thrust coefficient remains roughly constant in function of velocity with a value of $C_{T}=0.75 \pm 0.04$. The related distribution of the thrust coefficient over the different models is shown in figure 15, for velocities between $7.5<U<12 \mathrm{~m} / \mathrm{s}$. Since during the experiments, the lowest mean hub-height velocity was $8 \mathrm{~m} / \mathrm{s}$, and the porous disk models operate in a very turbulent boundary layer, we assume the thrust coefficient to be Reynolds independent for the considered range of velocities.

From the static calibration it became clear that an extra measurement uncertainty is introduced by the creep of the material when a force is applied over a long time. Special care was taken during the design to minimize the amount of creep that the models show. The ABS polymer was found to give the best material properties. The amount of creep increases with the amount of strain. An optimal stiffness was determined for the lowest amount of creep while still maintaining 
enough measurement precision. Moreover, creep is linearly corrected with the measured strain at the end of the measurement, when the strain should be zero. This corrections is typically on the order of $4 \%$.

The uncertainty of the reconstructed signals was estimated from an error propagation analysis resulting in: $\delta U_{i} / U_{1}= \pm 0.03$ and $\delta P_{i} / P_{1}= \pm 0.08$.

\section{Results and discussion}

The measurement capabilities of the designed porous disk model are demonstrated with wind tunnel measurements of a micro wind farm with 100 porous disk models. The measurement set-up is described in $\S 3.1$. The mean row power and unsteady loading are discussed as a function of the wind farm layout in $§ 3.2$. The spatio-temporal measurement capabilities are verified by studying the cross correlations between various turbine models in the wind farm in $§ 3.3$.

\subsection{Wind farm set-up}

A scaled down wind farm with 100 porous disk models was installed in the Corrsin Wind Tunnel. The wind farm consists of 20 rows and 5 columns with a spacing of $s_{x} / D=7$ and $s_{y} / D=5$. The sixty porous disk models in the central three columns were selected for the strain gage measurements, to use the instrumentation resources on those models least affected by wind farm border effects (figure 3 ). The remaining models were used for the two side columns, and were not measured during experiments. A schematic overview of the wind farm measurement set-up is shown on figure 2 . The first half of the wind tunnel is used to develop the boundary layer naturally, after being tripped by chains at the entrance of the test section.

The boundary layer has a height of $\delta_{99}=0.16 \mathrm{~m}$ when it reaches the model wind farm, which corresponds to 4 times the porous disk top-height. Figure 16 shows the measured flow characteristics of the boundary layer when it reaches the wind farm. The roughness length is determined by extrapolating the log-law velocity profile to the zero velocity at the wall. The measured roughness length is $z_{0}=0.9 \times 10^{-2} \mathrm{~mm}$, which with a scaling of 1:3333 corresponds to a full scale value of $z_{0}=0.03 \mathrm{~m}$. This corresponds to a moderately rough boundary layer $[52,16,13]$. The measured friction velocity, calculated from the slope of the log-law velocity profile and a Von Kármán constant of $\kappa=0.4$, is found to be $u_{\tau}=0.6 \mathrm{~m} / \mathrm{s}$. Scaled to full scale, the representative boundary layer height of this experiment is approximately $500 \mathrm{~m}$, for a scaling ratio of 1:3333. This falls in

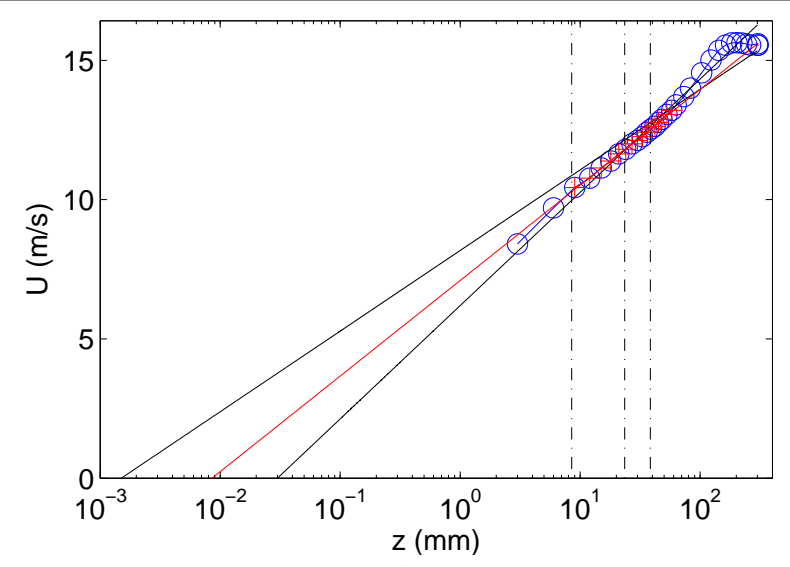

(a)

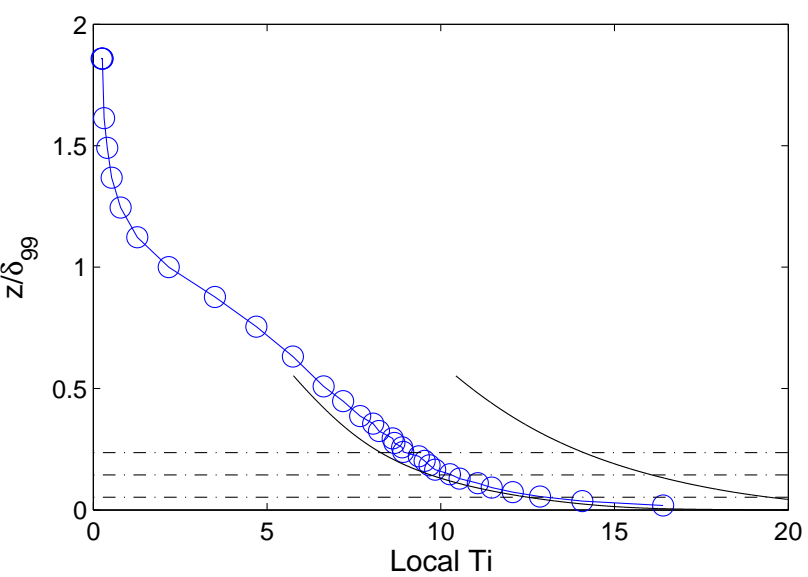

(b)

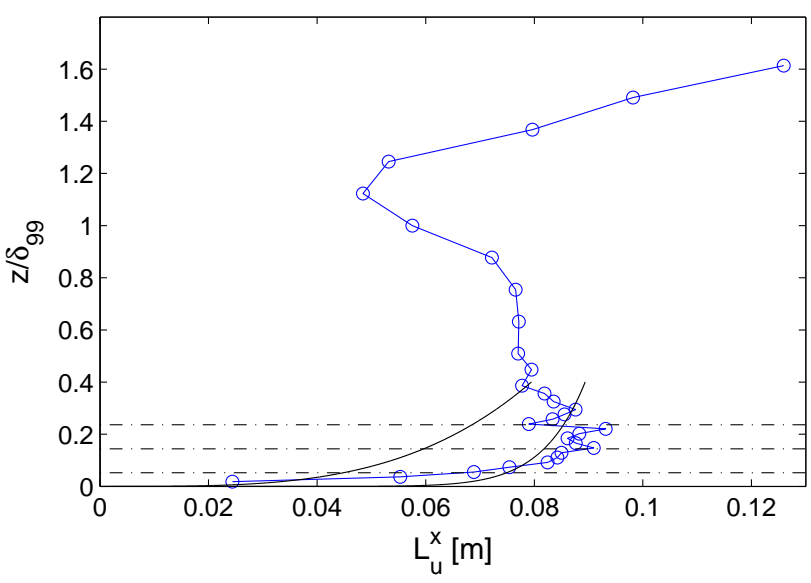

(c)

Fig. 16 Mean velocity (a), local streamwise turbulence intensity (b) and streamwise integral length scale (c) of the incoming boundary layer, measured with a 1-component hotwire probe. The red crosses on the velocity profile indicate the selected measurement points for fitting the log-law velocity profile. Dashed lines represent the porous disk top-, bottom- and hub- height. Black lines represent the range of profiles for a slightly rough boundary layer according to the VDI-guideline 3783/12 [52] and the ESDU guidelines [16] and (c) the range of integral length scales for a neutral ABL, according to Counihan et al. [13] 


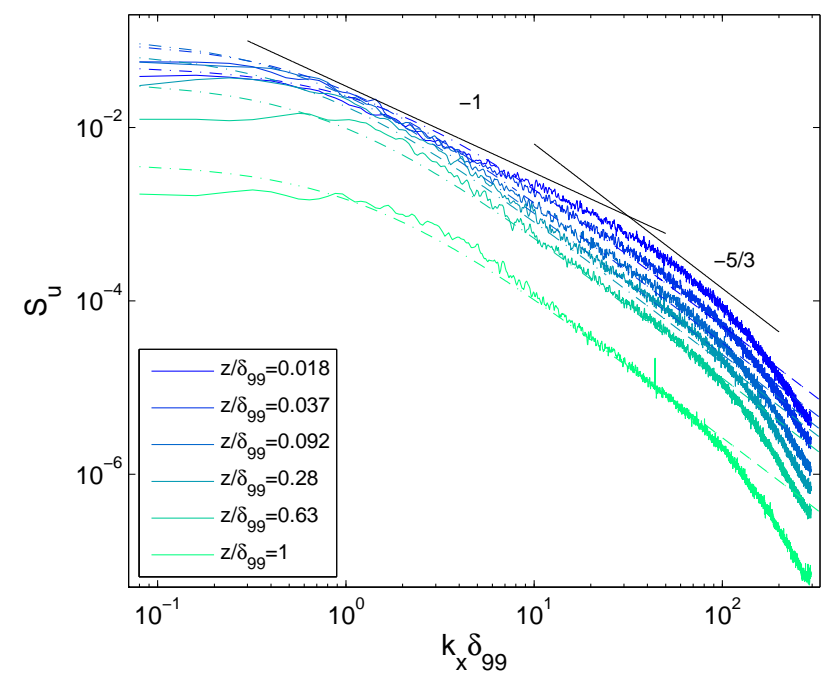

Fig. 17 Spectral density of the streamwise velocity component, measured with a 1-component hot-wire probe, and for different heights in the boundary layer. The dashed lines show the Kaimal spectrum based on the measured integral length scales [8].

the lower part of the range that can be expected for a neutrally moderately rough boundary layer: approximately $400 \mathrm{~m}$ to $1500 \mathrm{~m}[51,22,23,42]$. Moreover, from figure 16 it is seen that the porous disk models are located well within the log region of the velocity profile. A larger boundary layer thickness would result in a further extended log region with the same scaling of the velocity, so that the turbines would effectively experience the same profile.

The measured velocity profile, local streamwise turbulence intensity and integral length scale are shown on figure 16 together with the suggested profiles for a moderately rough neutral atmospheric boundary layer (ABL), according to the VDI-guideline 3783/12 [52] and the ESDU guidelines [16]. The streamwise local turbulence intensity at hub height is approximately $10 \%$. The integral length scale is calculated from the point where the autocorrelation reaches a value of $e^{-1}$, and by applying Taylor's hypothesis. The measured integral length scales at hub height are approximately three times larger than the porous disk's diameter and are of the same order of magnitude as the profiles suggested for a scaled moderately rough boundary layer [13]. The integral length scale in the boundary layer is important for a realistic amount of wake meandering in the wind farm, as this phenomena has been connected to the larger scales in the boundary layer [17].

Figure 17 shows the spectral density of the streamwise velocity component, for several heights in the boundary layer $z / \delta_{99}$. Close to the wall a -1 and $-5 / 3$ power law range are observed, depending on the height. In the log-layer, the spectra show a relatively good agreement with the Kaimal model, given by $S u(k)=4 \sigma_{u}^{2}(L / \bar{U}) /$ $(1+6 k L / \bar{U})^{5 / 3}$. The length scale $L$ is defined by matching the high wave number asymptote with the von Karman spectrum leading to $L=2.329 L_{u}^{x}$ [8], and making use of the measured integral length scale $L_{u}^{x}$.

Measurements are done for six different wind farm layouts. The first layout is the classical aligned layout. Five more layouts are considered by shifting the even rows in steps of $0.5 \mathrm{D}$ in the spanwise direction, until a staggered layout is reached at a shift of $2.5 \mathrm{D}$.

Strain signals are measured with Omega iNET-423 voltage input cards in combination with one Omega iNET-430 16bit A/D converter. The internal 4kHz lowpass filters are used to reduce high frequency noise. The large number of simultaneous strain gage measurements limits the sampling frequency per model to $0.866 \mathrm{kHz}$, lower than advised by the Nyquist criteria for the available filter. However, measurements for a single model have shown that the aliasing error is small for the frequency range of interest: $0-200 \mathrm{~Hz}$. The measurement time for each layout was between 5 to 15 minutes, over 3 to $9 \times 10^{4}$ times the largest integral time scale $(\approx 9 \mathrm{~ms})$ measured for the incoming boundary layer, so that very well converged statistics are obtained.

\subsection{Mean power and unsteady loading}

Figure 18 shows the measured velocity, reconstructed power output and turbulence intensity for an aligned layout, calculated from the porous disk strain-measurements. Symbols indicate individual porous disk models.

The reconstructed power (figure 18(b)) is compared with data measured at the Horns Rev wind farm for a wind direction of $270 \pm 2^{\circ}$, a velocity of $8 \mathrm{~m} / \mathrm{s}$ and for neutral thermal stratification [6]. For this wind direction the wind turbine rows are aligned with the wind direction from west to east and $S_{x} / D=S_{y} / D=7$, while the columns are slightly angled to the perpendicular direction. Both cases show a similar power deficit and the same trend: the power decreases significantly after the first row, after which it stays constant.

It is important to consider the differences between both cases. Horns Rev operates in a slightly rough boundary layer with a turbulence level of $<8 \%$, while the experiment is done for a moderately rough boundary layer with a turbulence intensity of $10 \%$ at hub height. While a higher turbulence level can lead to a better wake recovery and a smaller power deficit, the higher roughness length and velocity shear for the moderately rough boundary layer can lead to a lower power output asymptote at the end of the wind farm [37]. It should 


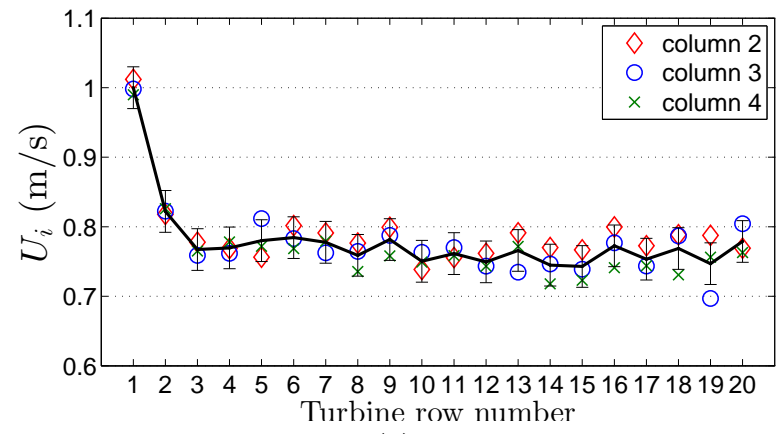

(a)

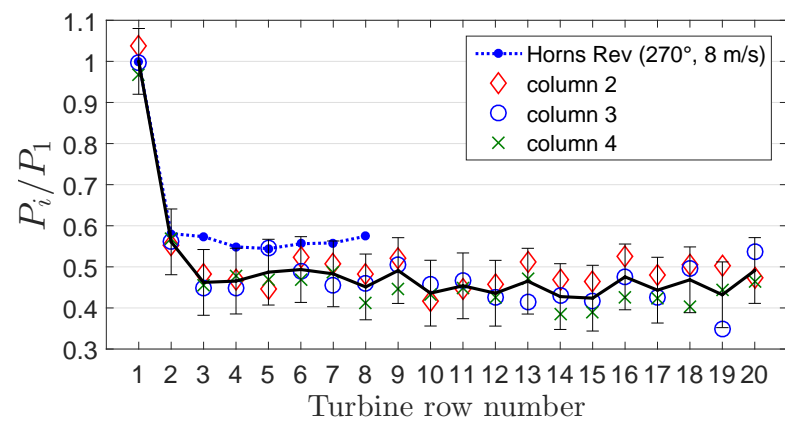

(b)

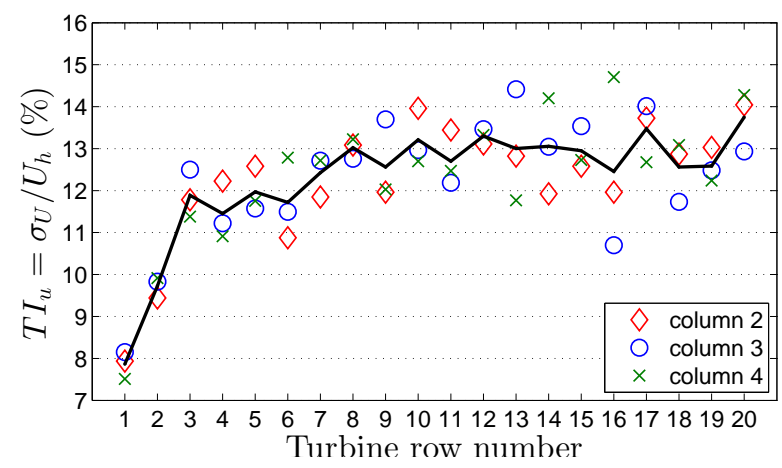

(c)

Fig. 18 Mean reconstructed velocity (a), mean reconstructed row power (b) and turbulence intensity (c), measured by the porous disk models, for an aligned wind farm.

also be noted that the spanwise spacing of the turbines in Horns Rev is $S_{y} / D=7$, while this spacing is smaller in the experiment: $S_{y} / D=5$.

Variations within each row are expected to be directly related to the measurement uncertainty and indicate the need to average over multiple models for each row.

Turbulence intensity is calculated from the reconstructed porous disk velocities and is therefore directly related to the unsteady loading of the wind turbines. As the measurements are limited to a frequency of up to $200 \mathrm{~Hz}$, the calculated turbulence intensity does not represent the total variance. Also the increase in turbu-

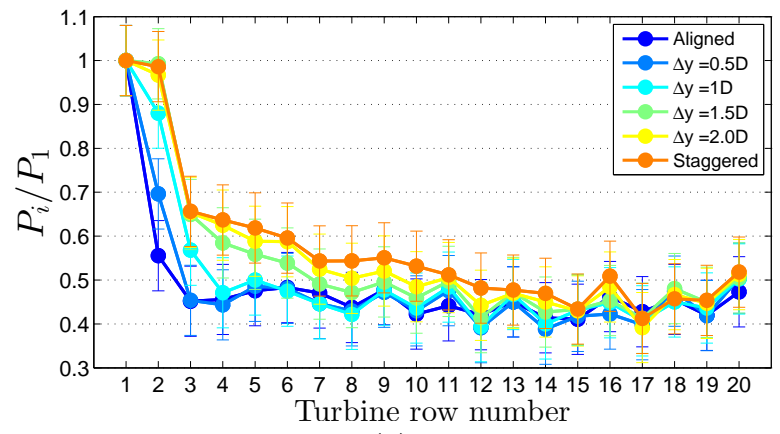

(a)

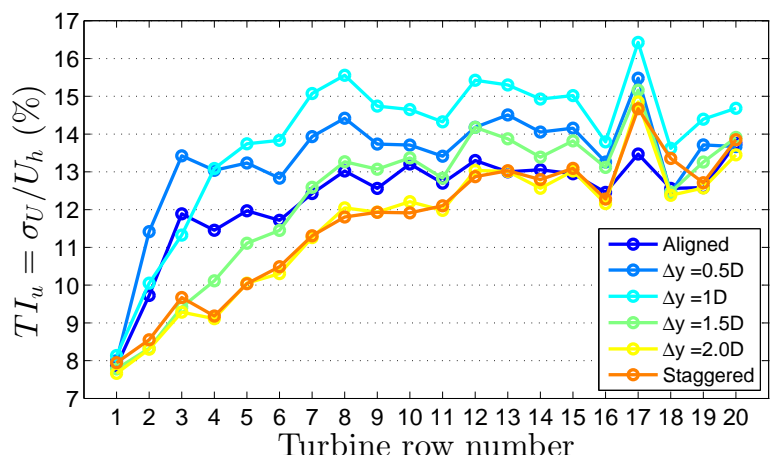

(b)

Fig. 19 Mean reconstructed row power and turbulence intensity as a function of the wind farm layout, measured by the porous disk models.

lence intensity levels off. However, only after approximately ten rows.

Figure 19 shows the reconstructed mean row power and turbulence intensity for the six different wind farm layouts. The change in mean row power shows a consistent trend in function of the wind farm layout. The most prominent improvements are seen for the first ten rows. For a staggered layout, the estimated power output is approximately equal for the first two rows, while the improvement at the end of the wind farm is limited. These trends are consistent with observations from Horns Rev for a wind direction of $263^{\circ}$ [4] and a LES simulation study [45]. By measuring the same trends as seen by a full scale wind farm, we conclude that the setup is useful to study differences in the mean row power between different layouts, within the limitations by the measurement uncertainty. To validate the absolute values of the power reduction a more detailed comparison is necessary with data for a wind farm with the exact same inflow conditions and wind farm characteristics. Averaging over all three models in each row is necessary to reduce the uncertainty. However, row 16 still shows a significant and consistent difference with its neighboring rows. This is expected to be caused by a systematic measurement error from a model in row 16, possibly 


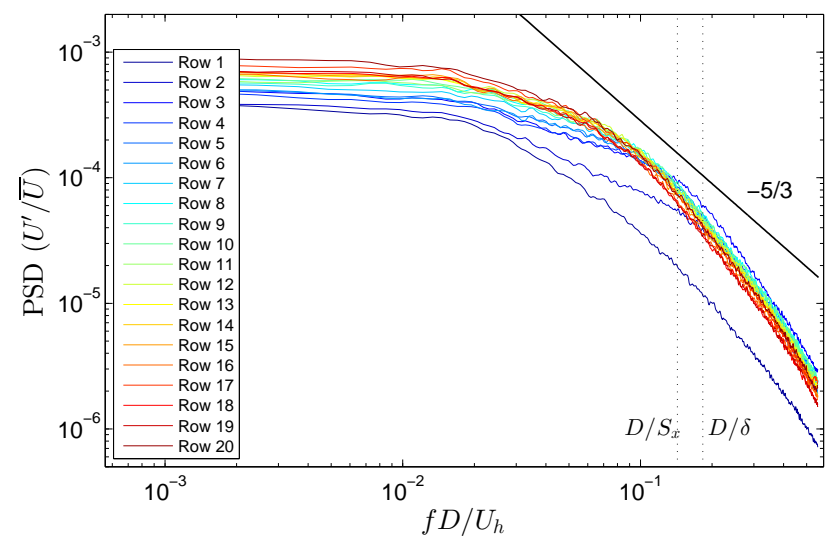

(a)

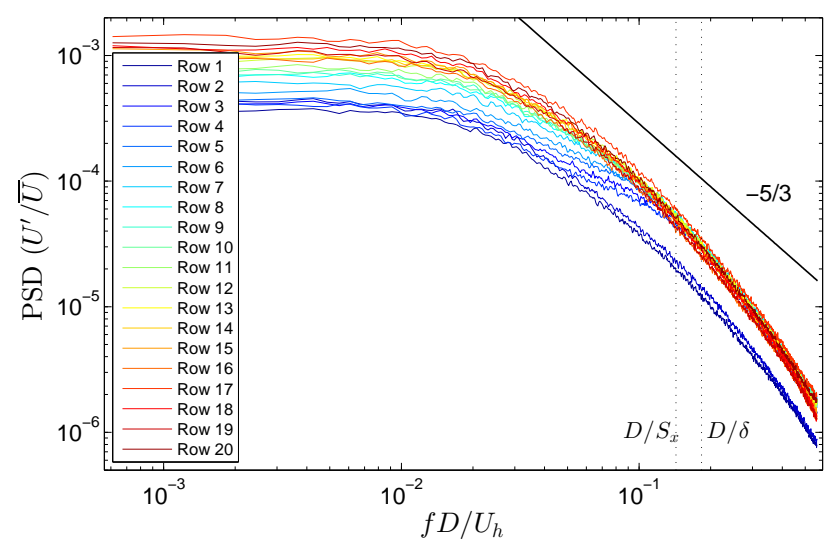

(b)

Fig. 20 Mean power spectral density of the measured reconstructed velocity signals by the porous disk models, for each row in the wind farm.

related to the calibration or a defect of the strain gage at the moment of measurement.

The turbulence intensity measurements show the highest unsteady loading for the intermediate layouts, in which the even rows are only slightly shifted: $0.5 D$ and $1 D$. The staggered layout results in the lowest unsteady loading, which is expected due to the largest turbine-to-turbine distance.

Figure 20 shows the power spectral density of the reconstructed velocity signals, measured by the porous disk models, for the aligned and the staggered layout. The spectra are calculated for each individual model and then averaged over each row. The spectrum of the first row can be considered as a measurement of the incoming flow with the application of a spatial filter. A power law behavior with a slope close to $-5 / 3$ is observed for the lower frequencies, consistent with the measurements in figure 17. For higher frequencies the slope becomes steeper due to the spatial filtering of the porous disk model.

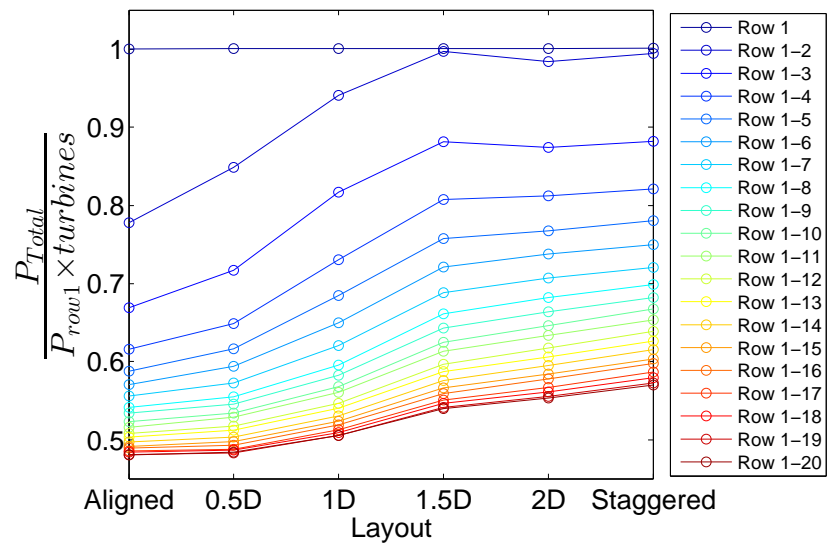

Fig. 21 Total reconstructed wind farm power as a function of wind farm layout and the number of rows.

For an aligned wind farm, the spectrum of the second row shows an increase in variance for a well defined frequency range, indicating the added turbulence by the upstream wake. A scaling for this frequency range is not obvious, however the corresponding length scale seems to be of the same order of magnitude as the streamwise wind farm spacing or the boundary layer height. The increase in variance for the third row of the staggered wind farm is observed at approximately the same frequency range, independent of the difference in wind farm layout. For more downstream rows the increased variance spreads over a larger range of scales.

Figure 21 shows the influence of the layout and the number of rows on the total wind farm power output. The output is normalized by the total power for a wakeless wind farm ${ }^{1}$. The influence of the layout on the mean row power is the most significant in the first rows of the wind farm. Considering more rows, hence reduces the difference in total power between an aligned and staggered case. However, for a wind farm with twenty rows, the staggered layout still results in a $6 \%$ higher power output than the aligned layout. This figure shows the same trend and a good agreement with the results from a similar LES study [45]. The absolute values for the power output are slightly lower for the experiments compared to the LES results. This can be explained by the differences between both studies, for example the slightly larger wind farm spacing and smaller roughness length in the LES study.

\subsection{Spatio-temporal correlations}

The power output of streamwise aligned turbines is known to be significantly correlated [47]. An improved understanding of this correlation can help explain and

1 A wind farm with all turbines operating in the front row. 
quantify the reduction in power fluctuations that is obtained after averaging over multiple turbines. The setup's capabilities to study these spatio-temporal characteristics are used for analysis of the turbine-to-turbine cross correlation of the power output. Figure 22 shows the correlation coefficients for the power output of every model in the three central columns with the power output of the central model in the first row, as a function of time. Each column is given a different color to visualize the correlation in the spanwise direction.

The results shown in figure 22(a), for the aligned layout, confirm a strong correlation with a corresponding time delay in the streamwise direction and small correlations in the spanwise direction. The time delay between peaks in high correlation is representative for the convective travel time of the velocity fluctuations between rows. The streamwise correlation is qualitatively significant up to ten rows.

Figure 22 (a) can be compared qualitatively with figure 8 (a) in reference [47] obtained from LES, showing very good agreement. This good agreement confirms the set-up's capabilities for studying features of the spatio-temporal characteristics of wind farms.

As shown in figure 22(b), shifting the even rows reduces the correlation for the shifted models, as the spanwise distance increases. The aligned models see an increase in correlation, as there are now less models located directly upstream to de-correlate the flow. For the staggered layout, streamwise algined models show a significant correlation to the end of the wind farm, spanning ten consecutive rows. Staggered models on the other hand, show an anti-correlation over a small number of rows.

A strong streamwise correlation of the streamwise velocity component and a smaller anti-correlation $(r \approx$ -0.2 ) have been measured before for a turbulent boundary layer [25]. These characteristics have been connected to the presence of long meandering features in the logarithmic region of a turbulent boundary layer. In [25], the spanwise location of the anti-correlation was found to be close to half the boundary layer thickness, at a height between $z / \delta=0.15-0.5$ in the turbulent boundary layer. The spanwise spacing for the staggered wind farm layout is $2.5 D=0.075 \mathrm{~m}$. This is indeed approximately half of the boundary layer height $\delta / 2=0.08 \mathrm{~m}$. We conclude that the measured correlations show a good agreement with the observations made for a turbulent boundary layer [25]. In a future study it should be studied how the presence of wind turbines or porous disk models in a boundary layer influence the correlation or de-correlation of the flow.

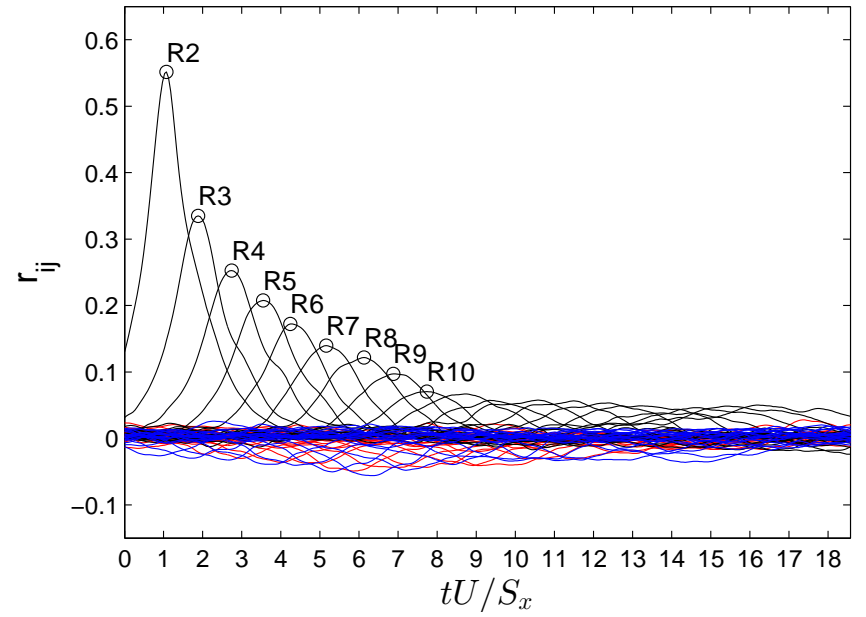

(a)

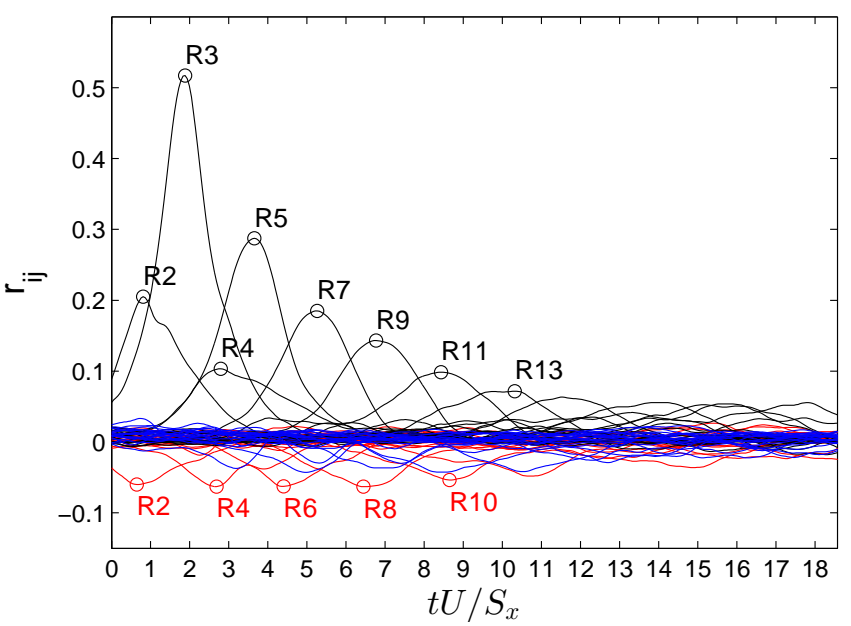

(b)

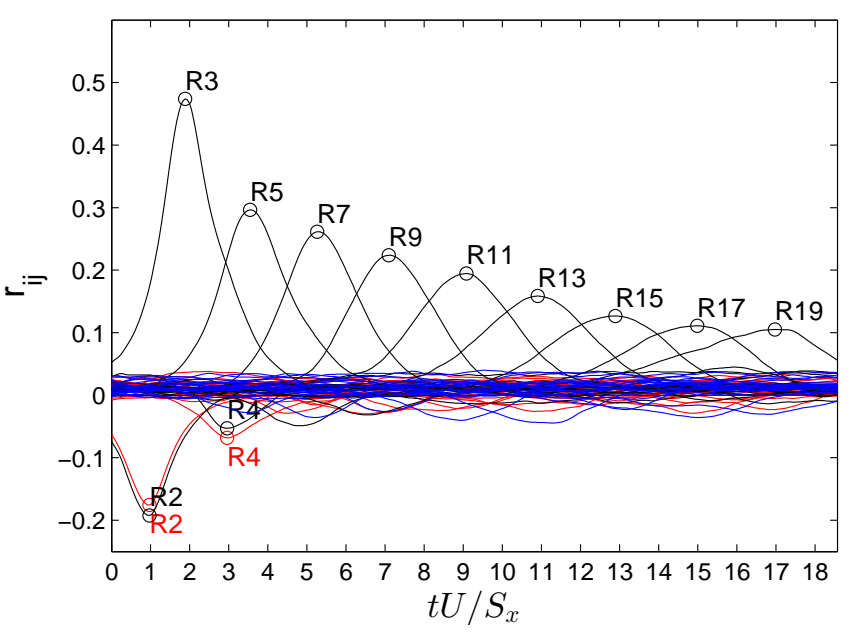

(c)

Fig. 22 Cross-correlation of the reconstructed power output of every porous disk with the porous disk in the first row and center column, for an aligned layout (a), a $1 D$ spanwise shift of the even rows (b) and a staggered layout (c). Colors indicate the different columns, according to figure 3 . Time is normalised by the streamwise model spacing $S_{x}$ and the mean velocity measured by the models in the first row. 


\section{Conclusions}

An experimental wind tunnel study of spatio-temporal characteristics of a model wind farm has been carried out. The experiment contains 100 porous disk models of wind turbines arranged in a $5 \times 20$ regular array. The 60 model turbines in the central three columns are instrumented with strain gages from which time histories of the thrust force have been acquired. The measured thrust signals were processed to deduce time histories of velocity and power. Making use of a model for the structural response, the measurement frequency reaches up to $200 \mathrm{~Hz}$. This corresponds to a reduced frequency of 0.6 when normalized with the mean hub velocity and disk diameter, or frequencies of $0.06 \mathrm{~Hz}$ when scaled to full-scale field conditions.

Time histories of power have been measured for six different wind farm layouts. Although the main focus of the acquisition technique is measuring the temporal characteristics, comparison of the measured mean row power has shown a reasonably good agreement with results for the Horns Rev wind farm [5]. The power drop for the aligned layout is approximately 50\%. The staggered layout shows an increased power output, with the largest increase for the first ten rows.

Total wind farm power has been studied as a function of the wind farm layout and the number of rows. For a wind farm with twenty rows, the staggered layout yields $6 \%$ higher power output than the aligned case. Shifting the even rows slightly such that porous disk models are located half in the wake of the upstream models, results in the highest unsteady loading. The staggered layout results in the lowest unsteady loading. It is concluded that the experimental setup of a model wind farm is able to capture the main trends in mean row power and unsteady loading, making it useful for layout optimization studies in the future.

The model's capabilities for measuring the spatiotemporal characteristics have been used to study the cross-correlation of the power outputs. Streamwise aligned porous disk models show a high correlation, confirming previous results by Stevens from an LES study [47]. An anti-correlation was observed for staggered porous disk models, showing a good agreement with measurement results for a turbulent boundary layer [25].

Acknowledgements The authors would like to thank Adrien Thormann for his contributions to the hot-wire measurements. Work supported by ERC (grant no. 306471, the ActiveWindFarms project) and by NSF (grants CBET-113380 and OISE1243482 , the WINDINSPIRE project).

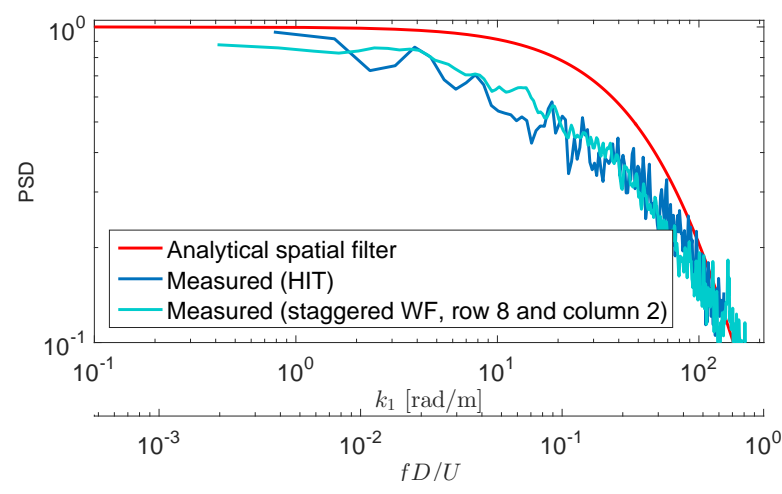

Fig. 23 Power spectral density of the spatial filtering by the porous disk for a measurement in homogeneous isotropic turbulence (HIT) generated with an active grid (cf. §2.2) and for a porous disk model in the wind farm, compared to the analytically described filter.

\section{Appendix}

We further investigate the effect of spatial filtering by the porous disk on the reconstructed velocity spectrum. First, we linearize the bending moment-velocity relation. Inertial effects on the thrust force can be neglected for frequencies significantly lower than the natural frequency, in our case lower than approximately $100 \mathrm{~Hz}$. For this frequency range, or by correcting with the structural model from figure 9, the bending moment-velocity-relation can be approximated by making use of axial-momentum theory:

$M(t)=\frac{1}{2} \rho C_{T} \frac{1}{(1-a)^{2}} \int_{A} U_{d}^{2}(y, z, t) z d A$,

where the bending moment-velocity relation is here expressed in terms of the local disk velocity $U_{d}(y, z, t)=U(y, z, t) /(1-a)$, with the axial induction factor $a$ defined from axial-momentum theory as $a=\left(1-\left(1-C_{T}\right)^{1 / 2}\right) / 2$ and $U(y, z, t)$ the free stream velocity. The velocity is then decomposed spatially:

$U_{d}(y, z, t)=\left\langle U_{d}\right\rangle(t)+\widetilde{U}_{d}(y, z, t)$,

and temporally:

$\left\langle U_{d}\right\rangle(t)=\left\langle\bar{U}_{d}\right\rangle+\left\langle U_{d}\right\rangle^{\prime}(t)$

$\widetilde{U}_{d}(y, z, t)=\overline{\widetilde{U}}_{d}(y, z)+\widetilde{U}_{d}^{\prime}(y, z, t)$.

By neglecting the higher order terms, the bending momentvelocity relation for the moment fluctuations can be linearized,

$\frac{M^{\prime}(t)(1-a)^{2}}{\frac{1}{2} \rho C_{T} A} \approx 2\left\langle\bar{U}_{d}\right\rangle\left\langle U_{d}\right\rangle^{\prime}\langle z\rangle$

and defined in terms of the incoming free-stream velocity fluctuations $\langle U\rangle^{\prime}(y, z, t)$ by making use of the relation for the induction factor.

$\langle U\rangle^{\prime}(t) \approx \frac{M^{\prime}(t)}{\rho C_{T} A\langle\bar{U}\rangle\langle z\rangle}$

To model the effect of spatial filtering, we follow the approach by Wyngaard [55] for spatial filtering by hot-wire probes and Lavoie [29] for spatial filtering effects of particle image velocimetry (PIV). The unfiltered $\left(E_{11}\right)$ and filtered $\left(E_{11}^{<}\right)$one-dimensional energy spectrum of the velocity fluctuations are defined by the integration of the unfiltered $\left(\Phi_{11}\right)$ 
and filtered $\left(\Phi_{11}^{<}\right)$velocity-spectrum tensor over all transverse wave numbers $k_{2}$ and $k_{3}$.

$E_{11}\left(k_{1}\right)=2 \iint_{-\infty}^{\infty} \Phi_{11}(\mathbf{k}) d k_{2} d k_{3}$

$E_{11}^{<}\left(k_{1}\right)=2 \iint_{-\infty}^{\infty} \Phi_{11}^{<}(\mathbf{k}) d k_{2} d k_{3}$,

The spatial filter of the porous disk can be described by a radial box filter with a diameter $\Delta$ in physical space and defined by the sinc function in wavenumber space. The spatial filter is expressed in function of the radial wavenumber $k_{r}^{2}=k^{2}-k_{1}^{2}$ :

$A\left(k_{r}\right)=\frac{\sin \left(k_{r} \Delta / 2\right)}{k_{r} \Delta / 2}$.

The one dimensional spectrum can be computed by assuming isotropic turbulence to model the velocity-spectral tensor:

$\Phi_{11}(\mathbf{k})=\frac{E(k)}{4 \pi k^{2}}\left(1-\frac{k_{1}^{2}}{k^{2}}\right)$,

multiplying by the filter's transfer function $A^{2}\left(k_{r}\right)$ and integrating:

$E_{11}^{<}\left(k_{1}\right)=\int_{0}^{\infty} A^{2}\left(k_{r}\right) \frac{E(k)}{k^{2}}\left(1-\frac{k_{1}^{2}}{k^{2}}\right) k_{r} d k_{r}$.

For the energy-spectrum function $E(k)$, a Kolmogorov spectrum $E(k)=C \epsilon^{2 / 3} k^{-5 / 3}$ is assumed:

$E_{11}^{<}\left(k_{1}\right)=C \epsilon^{2 / 3} \int_{0}^{\infty}\left(\frac{\sin \left(k_{r} \Delta / 2\right)}{k_{r} \Delta / 2}\right)^{2}\left(k_{1}^{2}+k_{r}^{2}\right)^{-17 / 6} k_{r}^{3} d k_{r}$

The unfiltered spectrum is given by:

$E_{11}\left(k_{1}\right)=\frac{18}{55} C \epsilon^{2 / 3} k_{1}^{-5 / 3}$.

By numerical integration, the filter transfer function can be computed:

$r_{u}\left(k_{1}\right)=\frac{E_{11}^{<}\left(k_{1}\right)}{E_{11}\left(k_{1}\right)}$

In figure 23 the numerically integrated transfer function $r_{u}\left(k_{1}\right)$, shown by the top solid line in red, is compared with the ratio of the hot-wire signal and the reconstructed velocity from the porous disk strain-gage signal. It is observed that the analytical spatial filter does not perfectly describe the measured ratio. For intermediate wave numbers (between 5$70 \mathrm{rad} / \mathrm{m}$ ), differences of up to $40 \%$ are observed for the power spectrum. Possible reasons for this difference are the approximations in the filter model, i.e. the assumption of isotropic turbulence, a radial box filter for the porous disk and possible nonlinear effects that are not taken into account by the linearization in Eq. (8).

\section{References}

1. Aho, J., Buckspan, A., Laks, J., Fleming, P., Jeong, Y., Dunne, F., Churchfield, M., Pao, L., Johnson, K.: A tutorial of wind turbine control for supporting grid frequency through active power control. In: American Control Conference (ACC), 2012, pp. 3120-3131. IEEE (2012)
2. Apt, J.: The spectrum of power from wind turbines. Journal of Power Sources 169(2), 369 - 374 (2007)

3. Aubrun, S., Loyer, S., Hancock, P., Hayden, P.: Wind turbine wake properties: Comparison between a nonrotating simplified wind turbine model and a rotating model. Journal of Wind Engineering and Industrial Aerodynamics 120, $1-8$ (2013)

4. Barthelmie, R.J., Frandsen, S.T., Rathmann, O., Hansen, K.S., Politis, E., Prospathopoulos, J., Schepers, J., Rados, K., Cabezón, D., Schlez, W., et al.: Flow and wakes in large wind farms: Final report for upwind wp8. Tech. rep., Danmarks Tekniske Universitet, Ris $\varnothing$ Nationallaboratoriet for Bæredygtig Energi (2011)

5. Barthelmie, R.J., Hansen, K., Frandsen, S.T., Rathmann, O., Schepers, J., Schlez, W., Phillips, J., Rados, K., Zervos, A., Politis, E., et al.: Modelling and measuring flow and wind turbine wakes in large wind farms offshore. Wind Energy 12(5), 431-444 (2009)

6. Barthelmie, R.J., Rathmann, O., Frandsen, S.T., Hansen, K., Politis, E., Prospathopoulos, J., Rados, K., Cabezón, D., Schlez, W., Phillips, J., et al.: Modelling and measurements of wakes in large wind farms. In: Journal of Physics: Conference Series, vol. 75, p. 012049. IOP Publishing (2007)

7. Bartl, J., Pierella, F., Sætrana, L.: Wake measurements behind an array of two model wind turbines. Energy Procedia 24, 305-312 (2012)

8. Burton, T., Sharpe, D., Jenkins, N., Bossanyi, E.: Wind energy handbook. John Wiley \& Sons (2001)

9. Cal, R.B., Lebrón, J., Castillo, L., Kang, H.S., Meneveau, C.: Experimental study of the horizontally averaged flow structure in a model wind-turbine array boundary layer. Journal of Renewable and Sustainable Energy 2(1) (2010)

10. Camp, E.H., Cal, R.B.: Mean kinetic energy transport and event classification in a model wind turbine array versus an array of porous disks: Energy budget and octant analysis. Physical Review Fluids 1(4), 044,404 (2016)

11. Chamorro, L.P., Porté-Agel, F.: Effects of thermal stability and incoming boundary-layer flow characteristics on wind-turbine wakes: a wind-tunnel study. Boundarylayer meteorology 136(3), 515-533 (2010)

12. Chamorro, L.P., Porté-Agel, F.: Turbulent flow inside and above a wind farm: A wind-tunnel study. Energies 4(11), 1916 (2011)

13. Counihan, J.: Adiabatic atmospheric boundary layers: a review and analysis of data from the period 1880-1972. Atmospheric Environment (1967) 9(10), 871-905 (1975)

14. De Rijcke, S., Driesen, J., Meyers, J.: Power smoothing in large wind farms using optimal control of rotating kinetic energy reserves. Wind Energy 18(10) (2015)

15. Ebert, P.R., Wood, D.H.: The near wake of a model horizontal-axis wind turbine - I. Experimental arrangements and initial results. Renewable Energy 12, 225 (1997)

16. ESDU: Characteristics of atmospheric turbulence near the ground, Part II: single point data for strong winds (neutral atmosphere). (1985)

17. España, G., Aubrun, S., Loyer, S., Devinant, P.: Spatial study of the wake meandering using modelled wind turbines in a wind tunnel. Wind Energy 14(7), 923-937 (2011)

18. Farr, T., Hancock, P.: Torque fluctuations caused by upstream mean flow and turbulence. In: Journal of Physics: Conference Series, vol. 555, p. 012048. IOP Publishing (2014) 
19. Fleming, P.A., Aho, J., Buckspan, A., Ela, E., Zhang, Y., Gevorgian, V., Scholbrock, A., Pao, L., Damiani, R.: Effects of power reserve control on wind turbine structural loading. Wind Energy (2015)

20. G. P. Corten, P.S., Hegberg, T.: Turbine interaction in large offshore wind farms. wind tunnel measurements. ECN report ECN-C-04-048 (2004)

21. Giberson, M.: Assessing wind power cost estimates. Tech. rep., Center for Energy Commerce, Texas Tech University (2013)

22. Grant, A.: Observations of boundary layer structure made during the 1981 kontur experiment. Quarterly Journal of the Royal Meteorological Society 112(473), 825-841 (1986)

23. Guo, J., Miao, Y., Zhang, Y., Liu, H., Li, Z., Zhang, W., He, J., Lou, M., Yan, Y., Bian, L., et al.: The climatology of planetary boundary layer height in china derived from radiosonde and reanalysis data. Atmos. Chem. Phys. Discuss., doi 10 (2016)

24. Hamilton, N., Kang, H.S., Meneveau, C., Cal, R.B.: Statistical analysis of kinetic energy entrainment in a model wind turbine array boundary layer. Journal of Renewable and Sustainable Energy 4(6), 063,105 (2012)

25. Hutchins, N., Marusic, I.: Evidence of very long meandering features in the logarithmic region of turbulent boundary layers. Journal of Fluid Mechanics 579, 1-28 (2007)

26. Hutchins, N., Nickels, T.B., Marusic, I., Chong, M.: Hotwire spatial resolution issues in wall-bounded turbulence. Journal of Fluid Mechanics 635, 103-136 (2009)

27. IRENA: Renewable energy technologies: cost analysis series. Tech. Rep. Volume 1: Power Sector - Wind Power (2012)

28. Kang, H., Meneveau, C.: Direct mechanical torque sensor for model wind turbines. Meas. Sci. Technol. 21, 105,206 (2010)

29. Lavoie, P., Avallone, G., De Gregorio, F., Romano, G., Antonia, R.: Spatial resolution of piv for the measurement of turbulence. Experiments in Fluids 43(1), 39-51 (2007)

30. Lebrón, J., Cal, R.B., Kang, H.S., Castillo, L., Meneveau, C.: Interaction between a wind turbine array and a turbulent boundary layer. 11th Americas Conference on Wind Engineering (2009)

31. Lebrón, J., Castillo, L., Meneveau, C.: Experimental study of the kinetic energy budget in a wind turbine streamtube. Journal of Turbulence (13), N43 (2012)

32. Lignarolo, L., Ragni, D., van Bussel, G., et al.: Kinetic energy entrainment in wind turbine and actuator disc wakes: an experimental analysis. In: Journal of Physics: Conference Series, vol. 524, p. 012163. IOP Publishing (2014)

33. Lignarolo, L., Ragni, D., Krishnaswami, C., Chen, Q., Ferreira, C.S., Van Bussel, G.: Experimental analysis of the wake of a horizontal-axis wind-turbine model. Renewable Energy 70, 31-46 (2014)

34. Lignarolo, L., Ragni, D., Simao Ferreira, C., van Bussel, G.J.: Wind turbine and actuator disc wake: two experimental campaigns. In: Proceedings of the 14th International Conference on Wind Engineering, ICWE14, Porto Alegre (Brasil), June 21-26, 2015. UFRGS (2015)

35. Maeda, T., Kamada, Y., Murata, J., Yonekura, S., Ito, T., Okawa, A., Kogaki, T.: Wind tunnel study on wind and turbulence intensity profiles in wind turbine wake. Journal of Thermal Science 20(2), 127-132 (2011)

36. Medici, D., Alfredsson, P.H.: Measurements on a wind turbine wake: $3 \mathrm{~d}$ effects and bluff body vortex shedding. Wind Energy 9(3), 219-236 (2006)
37. Meneveau, C.: The top-down model of wind farm boundary layers and its applications. Journal of Turbulence 13(7), 1-12 (2012)

38. Meyers, J., Meneveau, C.: Optimal turbine spacing in fully developed wind farm boundary layers. Wind Energy 15, 305-317 (2012)

39. Milborrow, D.: The performance of arrays of wind turbines. Journal of Industrial Aerodynamics 5, 403-430 (1980)

40. Mohamed, M.S., LaRue, J.C.: The decay power law in grid-generated turbulence. Journal of Fluid Mechanics 219, 195-214 (1990)

41. Odemark, Y.: Wakes behind wind turbines-studies on tip vortex evolution and stability (2012)

42. Seidel, D.J., Zhang, Y., Beljaars, A., Golaz, J.C., Jacobson, A.R., Medeiros, B.: Climatology of the planetary boundary layer over the continental united states and europe. Journal of Geophysical Research: Atmospheres 117(D17) (2012)

43. Simmons, R.T., Yonk, R.M., Hansen, M.E.: The true cost of energy: Wind. Tech. rep., STRATA, Institute of Political Economy (2015)

44. Sørensen, P., Hansen, A.D., Rosas, P.A.C.: Wind models for simulation of power fluctuations from wind farms. Journal of wind engineering and industrial aerodynamics 90(12), 1381-1402 (2002)

45. Stevens, R.J.A.M., Gayme, D.F., Meneveau, C.: Large eddy simulation studies of the effects of alignment and wind farm length. Journal of Renewable and Sustainable Energy 6(2) (2014)

46. Stevens, R.J.A.M., Graham, J., Meneveau, C.: A concurrent precursor inflow method for Large Eddy Simulations and applications to finite length wind farms. Renewable energy 68, 46-50 (2014)

47. Stevens, R.J.A.M., Meneveau, C.: Temporal structure of aggregate power fluctuations in large-eddy simulations of extended wind-farms. Journal of Renewable and Sustainable Energy 6(4) (2014)

48. Theunissen, R., Housley, P., Allen, C.B., Carey, C.: Experimental verification of computational predictions in power generation variation with layout of offshore wind farms. Wind Energy 18(10) (2015)

49. Thormann, A., Meneveau, C.: Decay of homogeneous, nearly isotropic turbulence behind active fractal grids. Physics of Fluids (1994-present) 26(2), 025,112 (2014)

50. Thormann, A., Meneveau, C.: Decaying turbulence in the presence of a shearless uniform kinetic energy gradient. Journal of Turbulence 16(5), 442-459 (2015)

51. Tjernstrm, M., Smedman, A.S.: The vertical turbulence structure of the coastal marine atmospheric boundary layer. Journal of Geophysical Research: Oceans 98(C3), 4809-4826 (1993). DOI 10.1029/92JC02610. URL http://dx.doi.org/10.1029/92JC02610

52. VDI-guideline 3783/12: Environmental meteorology physical modelling of flow and dispersion processes in the atmospheric boundary layer, applications of wind tunnels. (2000)

53. Whale, J., Anderson, C.G., Bareiss, R., Wagner, S.: An experimental and numerical study of the vortex structure in the wake of a wind turbine. J. Wind Eng. Ind. Aerodyn. 84, 1 (2000)

54. Wu, Y., Porté-Agel, F.: Large-eddy simulation of windturbine wakes: evaluation of turbine parametrizations. Boundary-Layer Meteorology 138(3), 345-366 (2011)

55. Wyngaard, J.: Measurement of small-scale turbulence structure with hot wires. Journal of Physics E: Scientific Instruments 1(11), 1105 (1968) 
56. Zhang, W., Markfort, C., Porté-Agel, F.: Near-wake flow structure downwind of a wind turbine in a turbulent boundary layer. Experiments in Fluids 52(5) (2012) 